\title{
Discrepancy of Cartesian Products of Arithmetic Progressions
}

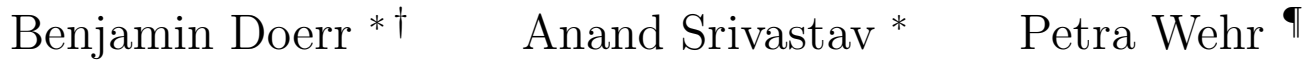 \\ — Dedicated to the memory of Walter Deuber —
}

Submitted: Jul 31, 2003; Accepted: Sep 3, 2003; Published: Jan 2, 2004

MR Subject Classifications: 11B25, 11K38, 22B05, 05C15

Keywords: arithmetic progressions, discrepancy, harmonic analysis, locally compact abelian groups.

\begin{abstract}
We determine the combinatorial discrepancy of the hypergraph $\mathcal{H}$ of cartesian products of $d$ arithmetic progressions in the $[N]^{d}$-lattice $([N]=\{0,1, \ldots, N-1\})$. The study of such higher dimensional arithmetic progressions is motivated by a multi-dimensional version of van der Waerden's theorem, namely the Gallai-theorem (1933). We solve the discrepancy problem for $d$-dimensional arithmetic progressions by proving $\operatorname{disc}(\mathcal{H})=\Theta\left(N^{\frac{d}{4}}\right)$ for every fixed integer $d \geq 1$. This extends the famous lower bound of $\Omega\left(N^{1 / 4}\right)$ of Roth (1964) and the matching upper bound $O\left(N^{1 / 4}\right)$ of Matoušek and Spencer (1996) from $d=1$ to arbitrary, fixed $d$. To establish the lower bound we use harmonic analysis on locally compact abelian groups. For the upper bound a product coloring arising from the theorem of Matoušek and Spencer is sufficient. We also regard some special cases, e.g., symmetric arithmetic progressions and infinite arithmetic progressions.
\end{abstract}

*Mathematisches Seminar II; Christian-Albrechts-Universität zu Kiel; Christian-Albrechts-Platz 4; 24098 Kiel; Germany; e-mail: \{bed,asr\}@numerik.uni-kiel.de

†Supported by the graduate school 'Effiziente Algorithmen und Multiskalenmethoden', Deutsche Forschungsgemeinschaft

`SAP AG Düsseldorf, e-mail: petra.wehr@sap-ag.de 


\section{Introduction}

Let $\mathcal{H}=(X, \mathcal{E})$ denote a hypergraph, i. e., $X$ is a finite set and $\mathcal{E}$ is a family of subsets of $X$. Let $\chi: X \rightarrow\{-1,+1\}$ be a 2 -coloring of $X$. For $E \in \mathcal{E}$ define $\chi(E)=\sum_{x \in E} \chi(x)$. The discrepancy of $\mathcal{H}$ is defined by

$$
\operatorname{disc}(\mathcal{H})=\min _{\chi} \max _{E \in \mathcal{E}}|\chi(E)|
$$

We are interested in arithmetic progressions in more than one dimension, but let us briefly review the one-dimensional case. Let $X=[N]=\{0, \ldots, N-1\}$ and let

$$
\mathcal{E}:=\{\{j, j+\delta, \ldots, j+l \delta\} \mid j, \delta, l \in[N], j+l \delta \in X\}
$$

denote the set of arithmetic progressions on $X$. The investigation of the discrepancy of the hypergraph $\mathcal{H}=(X, \mathcal{E})$ is an old issue in combinatorial discrepancy theory.

In 1927, van der Waerden proved [vdW27] that if the non-negative integers are colored with finitely many colors, then there is an arbitrarily long arithmetic progression in one color-class.

Investigating irregularities of arithmetic progressions, K. Roth [Rot64] exhibited another aspect of the same phenomenon: If we focus on long arithmetic progressions, then they might not be monochromatic but have large discrepancy. More precisely, he showed that $\operatorname{disc}(\mathcal{H})=\Omega\left(N^{\frac{1}{4}}\right)$. Roth himself did not believe that his lower bound is optimal, most probably due to the fact that the probabilistic method immediately gives the upper bound $O(\sqrt{N \log N})$. A. Sárközy [Sár74] was the first who improved the exponent of $N$ and showed an upper bound of $O\left(N^{\frac{1}{3}+o(1)}\right)$. A breakthrough was made by J. Beck in 1981 [Bec81], who showed that the lower bound is best possible up to a polylogarithmic factor by improving the upper bound to $O\left(N^{\frac{1}{4}} \log ^{\frac{5}{2}} N\right)$. It lasted 30 years until J. Matoušek and J. Spencer [MS96] finally solved the problem and proved that the upper bound is $O\left(N^{\frac{1}{4}}\right)$.

In this paper we focus on the discrepancy of higher dimensional arithmetic progressions, a problem posed by H. J. Prömel in 1996.

Definition 1.1. A d-dimensional arithmetic progression $A$ in $[N]^{d}$ is the cartesian product of $d$ arithmetic progressions in $[N], i$. e. $A=\prod_{i=1}^{d} A_{i}$ with $A_{i} \in \mathcal{E}$ for all $i=1, \ldots, d$.

The investigation of the discrepancy of such sets is motivated by Gallai's theorem [Rad33] (see also [GRS90]), which can be viewed as a kind of generalization of van der Waerden's theorem. By Gallai's theorem the following is true: Let $t$ be a positive integer and $V=[t]^{d}$. Then there exist integers $x_{1}, x_{2}, \ldots, x_{d}$ and $\delta \in \mathbb{N}$ such that

$$
W=\left\{\left(x_{1}+i_{1} \delta, x_{2}+i_{2} \delta, \ldots, x_{d}+i_{d} \delta\right): 0 \leq i_{j}<t, j=1, \ldots, d\right\}
$$


is monochromatic. Note that $W$ is a $d$-dimensional arithmetic progression in the sense of Definition 1.1.

The main result of this paper is

Theorem 1.2. Let $\mathcal{H}=\left([N]^{d}, \mathcal{E}\right)$ where $d \geq 1$ is an integer and $\mathcal{E}$ is the family of all $d$-dimensional arithmetic progressions in $[N]^{d}$. Then

$$
\pi^{-d} N^{\frac{d}{4}} \leq \operatorname{disc}(\mathcal{H}) \leq c^{d} N^{\frac{d}{4}}
$$

where $c$ is an absolute constant. Thus $\operatorname{disc}(\mathcal{H})=\Theta\left(N^{\frac{d}{4}}\right)$ for every fixed $d$.

Our proof of the lower bound is a variation of the Fourier transform method (in the literature also called circle-method). The novelty of our proof is the application of harmonic analysis on locally compact abelian groups, in particular the duality $\widehat{\mathbb{Z}} \simeq T:=\{z \in$ $\mathbb{C}|| z \mid=1\}$ and the direct use of the discrepancy function. This lower bound proof can also be found in Petra Wehr's dissertation [Weh97].

The upper bound follows easily by using the product of $d$ optimal colorings arising from the theorem of J. Matoušek and J. Spencer [MS96].

Some special cases are regarded as well, among them the following: A $d$-dimensional symmetric arithmetic progressions is the $d$-fold product of just one arithmetic progression. Here the upper bound for the discrepancy is as in the one-dimensional case $O\left(N^{\frac{1}{4}}\right)$ with a constant independent of $d$ and $N$. We conjecture that this is sharp apart from constant factors.

\section{The Lower Bound}

In this section, we determine the lower bound.

Theorem 2.1. Let $\mathcal{H}=\left([N]^{d}, \mathcal{E}\right)$, where $d \geq 1$ is an integer and $\mathcal{E}$ is the family of all $d$-dimensional arithmetic progressions in $[N]^{d}$. Then

$$
\operatorname{disc}(\mathcal{H}) \geq \pi^{-d} N^{\frac{d}{4}}
$$

Roth's proof of the lower bound in the one-dimensional case [Rot64] does not invoke the discrepancy function directly. This might be one reason why we were not able to generalize Roth's proof to higher dimensions. Instead we use a different approach (which in the case $d=1$ gives a new proof of Roth's theorem). As Roth's proof, our method is also a variation of the Fourier transform method. The novelty of our proof is the application of harmonic analysis on locally compact abelian groups, in particular the duality between $\widehat{\mathbb{Z}}$ 
and the torus $T=\{z \in \mathbb{C}|| z \mid=1\}$, and the representation of the discrepancy function as a convolution. It seems that our proof in the one dimensional case is more transparent than Roth's approach, although we use the abstract framework of locally compact abelian groups as described in Rudin's book [Rud62].

For the remainder of this paper let $d$ denote a positive integer. In this section we consider the group $G:=\mathbb{Z}^{d}$. Note that $G$ equipped with the discrete topology is a locally compact abelian group. A group-homomorphism $\gamma: G \rightarrow T$ is called a character, the set of characters of $G$ is denoted by $\widehat{G}$. The convolution of two functions $f, g \in L^{1}(G)$ is defined by $(f * g)(y):=\sum_{x \in G} f(x) g(y-x)$, the Fourier transform of $f$ is

$$
\widehat{f}: \widehat{G} \rightarrow \mathbb{C} ; \gamma \mapsto \sum_{x \in G} f(x) \gamma(-x)
$$

Note that we have $\widehat{f * g}=\widehat{f} \widehat{g}$. Let $<\cdot, \cdot>$ denote the inner product on $\mathbb{R}^{n}$. Using the duality $\widehat{\mathbb{Z}} \simeq T$ (see [Rud62]), it is straightforward to show the following proposition.

Proposition 2.2. For $\alpha \in\left[0,1^{d}\right.$ let $\gamma_{\alpha}: \mathbb{Z}^{d} \rightarrow T ; z \mapsto e^{2 \pi i<\alpha, z>}$ denote the character associated to $\alpha$ and $T^{d}:=\left\{\gamma_{\alpha} \mid \alpha \in\left[0,1\left[^{d}\right\}\right.\right.$.

(i) The dual group $\widehat{\mathbb{Z}^{d}}$ of $\mathbb{Z}^{d}$ is $T^{d}$.

(ii) The Fourier transform $\widehat{f}$ of a function $f \in L^{1}\left(\mathbb{Z}^{d}\right)$ can be written as

$$
\widehat{f}\left(\gamma_{\alpha}\right)=\sum_{z \in \mathbb{Z}^{d}} e^{-2 \pi i<\alpha, z>} f(z) .
$$

Proof of Theorem 2.1: Before going into details, let us sketch the proof idea. We express the discrepancy of a given $d$-dimensional arithmetic progression and a given 2-coloring as the convolution of the coloring function and a characteristic function of the arithmetic progression. Then we compute the $L^{2}$-norm of this function applying the Plancherel theorem for the group $G$. With an average argument (taking the sum over a special set of $d$-dimensional arithmetic progressions) and using an estimate for the sum of unit roots we are done.

We need some notation. Set

- $L:=\frac{1}{2} \sqrt{N}$,

- $\Delta:=\{1, \ldots, \sqrt{N}\}^{d}$,

- $J:=[N]^{d}$,

- $A_{j_{0}, \delta_{0}}:=\left\{j_{0}+\delta_{0} i \mid i \in[L]\right\} \cap[N]$, 
- For $\delta \in \Delta, j \in J$ define $A_{j, \delta}:=\prod_{i=1}^{d} A_{j_{i}, \delta_{i}}$.

Define the extension $\chi_{F}$ of a 2-coloring $\chi$ of $[N]^{d}$ to $\mathbb{Z}^{d}$ by

$$
\chi_{F}(j)=\left\{\begin{array}{rl}
\chi(j) & , \quad \text { if } j \in J \\
0 & , \quad \text { otherwise }
\end{array},\right.
$$

and define a (quasi-) characteristic function of $A_{0, \delta}$ by

$$
\eta_{\delta}(k)=\left\{\begin{array}{ll}
1, & \text { if }-k \in A_{0, \delta} \\
0, & \text { otherwise }
\end{array} .\right.
$$

An easy calculation then yields

$$
\left(\chi_{F} * \eta_{\delta}\right)(j)=\chi\left(A_{j, \delta}\right)
$$

for all $\delta \in \Delta, j \in J$.

As $\chi_{F}$ and $\eta_{\delta}$ have finite support, we have $\chi_{F} * \eta_{\delta} \in L^{1}\left(\mathbb{Z}^{d}\right) \cap L^{2}\left(\mathbb{Z}^{d}\right)$. The Plancherel theorem for locally compact abelian groups [Rud62] gives:

$$
\begin{aligned}
\sum_{j \in J} \chi^{2}\left(A_{j, \delta}\right) & \stackrel{(1)}{=}\left\|\chi_{F} * \eta_{\delta}\right\|_{2}^{2} \\
& =\left\|\widehat{\chi_{F} * \eta_{\delta}}\right\|_{2}^{2} \\
& =\left\|\widehat{\chi}_{F} \cdot \widehat{\eta}_{\delta}\right\|_{2}^{2} \\
& =\int_{[0,1]^{d}}\left|\widehat{\chi}_{F}\left(\gamma_{\alpha}\right) \widehat{\eta}_{\delta}\left(\gamma_{\alpha}\right)\right|^{2} d \alpha
\end{aligned}
$$

Roth [Rot64] showed the following estimate for sums of unit roots.

$$
\sum_{\delta=1}^{\sqrt{N}}\left|\sum_{j=0}^{L-1} e^{2 \pi i \delta j \alpha}\right|^{2} \geq \pi^{-2} N \quad \text { for arbitrary } \alpha \in \mathbb{R} .
$$


Thus we have

$$
\begin{aligned}
\sum_{\delta \in \Delta}\left|\widehat{\eta}_{\delta}\left(\gamma_{\alpha}\right)\right|^{2} & =\sum_{\delta \in \Delta}\left|\sum_{j \in \mathbb{Z}^{d}} \eta_{\delta}(j) e^{-2 \pi i<j, \alpha>}\right|^{2} \\
& =\sum_{\delta \in \Delta}\left|\sum_{j_{1}, \ldots, j_{d} \in[L]} e^{2 \pi i\left(j_{1} \delta_{1} \alpha_{1}+\cdots+j_{d} \delta_{d} \alpha_{d}\right)}\right|^{2} \\
& =\sum_{\delta \in \Delta}\left|\prod_{k=1}^{d}\left(\sum_{j_{k}=0}^{L-1} e^{2 \pi i j_{k} \delta_{k} \alpha_{k}}\right)\right|^{2} \\
& =\prod_{k=1}^{d}\left(\sum_{\delta_{k}=1}^{\sqrt{N}}\left|\sum_{j_{k}=0}^{L-1} e^{2 \pi i j_{k} \delta_{k} \alpha_{k}}\right|^{2}\right) \\
& \geq\left(\pi^{-2} N\right)^{d}=\pi^{-2 d} N^{d} .
\end{aligned}
$$

The Plancherel theorem yields

$$
\left\|\widehat{\chi}_{F}\right\|_{2}^{2}=\left\|\chi_{F}\right\|_{2}^{2}=\sum_{j \in J} \chi^{2}(j)=N^{d}
$$

Finally

$$
\begin{aligned}
\sum_{\delta \in \Delta} \sum_{j \in J} \chi^{2}\left(A_{j, \delta}\right) & \stackrel{(2)}{=} \sum_{\delta \in \Delta} \int_{[0,1]^{d}}\left|\widehat{\chi}_{F}\left(\gamma_{\alpha}\right) \widehat{\eta}_{\delta}\left(\gamma_{\alpha}\right)\right|^{2} d \alpha \\
& =\int_{[0,1]^{d}}\left|\widehat{\chi}_{F}\left(\gamma_{\alpha}\right)\right|^{2}\left(\sum_{\delta \in \Delta}\left|\widehat{\eta}_{\delta}\left(\gamma_{\alpha}\right)\right|^{2}\right) d \alpha \\
& \stackrel{(3)}{\geq}\left(\pi^{-2} N\right)^{d} \int_{[0,1]^{d}}\left|\widehat{\chi}_{F}\left(\gamma_{\alpha}\right)\right|^{2} d \alpha \\
& \stackrel{(4)}{=}\left(\pi^{-2} N\right)^{d} N^{d}=\pi^{-2 d} N^{2 d}
\end{aligned}
$$

The sum $\sum_{\delta \in \Delta} \sum_{j \in J} \chi^{2}\left(A_{j, \delta}\right)$ consists of $N^{\frac{3 d}{2}}$ terms. The pigeon-hole principle implies the existence of $\bar{\delta} \in \Delta$ and $\bar{j} \in J$ such that

$$
\chi^{2}\left(A_{\bar{j}, \bar{\delta}}\right) \geq \frac{\pi^{-2 d} N^{2 d}}{N^{\frac{3 d}{2}}}=\pi^{-2 d} N^{\frac{d}{2}} .
$$

So $\left|\chi\left(A_{\bar{j}, \bar{\delta}}\right)\right| \geq \pi^{-d} N^{\frac{d}{4}}$, and this means that the discrepancy of $\mathcal{H}=\left([N]^{d}, \mathcal{E}\right)$ is at least $\pi^{-d} N^{\frac{d}{4}}$. This establishes the lower bound. 


\section{The Upper Bound}

In this section we determine the upper bound for the discrepancy of $d$-dimensional arithmetic progressions:

Theorem 3.1. Let $\mathcal{H}$ be the hypergraph of d-dimensional arithmetic progressions in $[N]^{d}$. Then $\operatorname{disc}(\mathcal{H}) \leq c^{d} N^{\frac{d}{4}}$ for an absolute constant $c>0$.

We give a very general argument which solves the problem also for an arbitrary number of colors:

Let $\mathcal{G}=(X, \mathcal{E})$ and $\mathcal{H}=(Y, \mathcal{F})$ be hypergraphs. Define the direct product of $\mathcal{G}$ and $\mathcal{H}$ by

$$
\mathcal{G} \times \mathcal{H}:=(X \times Y,\{A \times B \mid A \in \mathcal{E}, B \in \mathcal{F}\}) .
$$

By this definition, the hypergraph of $d$-dimensional arithmetic progressions is the $d$-fold direct product of the hypergraph of (one-dimensional) arithmetic progressions on $[N]$.

Let us shortly introduce the notion of multi-color discrepancy (see also [DS03]). A $c^{-}$ coloring of $\mathcal{G}$ is a mapping $\chi: X \rightarrow M$, where $M$ is any set of cardinality $c$. For convenience, usually one has $M=[c]$. For a color $i \in[c]$ and a hyperedge $A \in \mathcal{E}$ the discrepancy of $A$ in color $i$ with respect to $\chi$ is defined by

$$
\operatorname{disc}_{\chi, i}(A):=|| \chi^{-1}(i) \cap A\left|-\frac{|A|}{c}\right|,
$$

which measures the deviation of the actual coloring from an (ideal) balanced coloring in respect to the color $i$. The discrepancy of $\mathcal{G}$ with respect to $\chi$ is

$$
\operatorname{disc}(\mathcal{G}, \chi):=\max _{i \in[c], A \in \mathcal{E}} \operatorname{disc}_{\chi, i}(A)
$$

and the discrepancy of $G$ in $c$ colors is

$$
\operatorname{disc}(\mathcal{G}, c):=\min _{\chi: X \rightarrow[c]} \operatorname{disc}(\mathcal{G}, \chi)
$$

Theorem 3.2. For any $c \in \mathbb{N}$ and any two hypergraphs $\mathcal{G}$ and $\mathcal{H}$ we have

$$
\operatorname{disc}(\mathcal{G} \times \mathcal{H}, c) \leq c \operatorname{disc}(\mathcal{G}, c) \operatorname{disc}(\mathcal{H}, c)
$$

Proof. Pick a Latin square $Q=\left(q_{i j}\right)$ of dimension $c$, i. e. $Q \in[c]^{[c] \times[c]}$ such that every row and column contains every number of $[c]$ exactly once. Note that for every $c \in \mathbb{N}$ there is a Latin square of dimension $c$ : Let $*$ be any group multiplication on $[c]$. Then $q_{i j}:=i * j$ defines a Latin square. As $Q$ is a Latin square we may define a permutation $\pi_{i}$ of $[c]$ for every $i \in[c]$ by the following rule: $\pi_{i}(j)$ is the unique $k \in[c]$ such that $q_{j k}=i$. 
Choose optimal colorings $\chi_{\mathcal{G}}$ and $\chi_{\mathcal{H}}$ of $\mathcal{G}$ and $\mathcal{H}$ respectively, i. e., $\operatorname{disc}\left(\mathcal{G}, \chi_{\mathcal{G}}\right)=\operatorname{disc}(\mathcal{G}, c)$ and $\operatorname{disc}\left(\mathcal{H}, \chi_{\mathcal{H}}\right)=\operatorname{disc}(\mathcal{H}, c)$. Define $\chi: X \times Y \rightarrow[c]$ by

$$
\chi(x, y):=q_{\chi_{\mathcal{G}}(x) \chi_{\mathcal{H}}(y)}
$$

for all $x \in X, y \in Y$.

Let $A \in \mathcal{E}, B \in \mathcal{F}$. Set

$$
\begin{aligned}
& a_{i}=\left|\chi_{\mathcal{G}}^{-1}(i) \cap A\right|-\frac{|A|}{c}, \\
& b_{i}=\left|\chi_{\mathcal{H}}^{-1}(i) \cap B\right|-\frac{|B|}{c}
\end{aligned}
$$

for all $i \in[c]$. Then we have

$$
\sum_{i=0}^{c-1} a_{i}=0=\sum_{i=0}^{c-1} b_{i}
$$

This yields

$$
\begin{aligned}
\left|\chi^{-1}(i) \cap(A \times B)\right| & =\sum_{j=0}^{c-1}\left|\chi_{G}^{-1}(j) \cap A\right|\left|\chi_{H}^{-1}\left(\pi_{i}(j)\right) \cap B\right| \\
& =\sum_{j=0}^{c-1}\left(a_{j}+\frac{|B|}{c}\right)\left(b_{\pi_{i}(j)}+\frac{|A|}{c}\right) \\
& =\sum_{j=0}^{c-1} a_{j} b_{\pi_{i}(j)}+\frac{|A|}{c} \sum_{j=0}^{c-1} a_{j}+\frac{|B|}{c} \sum_{j=0}^{c-1} b_{j}+c \frac{|A||B|}{c^{2}} \\
& =\sum_{j=0}^{c-1} a_{j} b_{\pi_{i}(j)}+\frac{|A \times B|}{c} \quad \text { by (5). }
\end{aligned}
$$

As $\left|a_{i}\right| \leq \operatorname{disc}(\mathcal{G}, c)$ and $\left|b_{i}\right| \leq \operatorname{disc}(\mathcal{H}, c)$, we have

$$
|| \chi^{-1}(i) \cap(A \times B)\left|-\frac{|A \times B|}{c}\right|=\sum_{j=0}^{c-1} a_{j} b_{\pi_{i}(j)} \leq c \operatorname{disc}(\mathcal{G}, c) \operatorname{disc}(\mathcal{H}, c) .
$$

This proves the theorem.

For two colors, this discrepancy notion nearly coincides with the one introduced in the beginning of this article: We have $\operatorname{disc}(\mathcal{G}, 2)=2 \operatorname{disc}(\mathcal{G})$. 
Theorem 3.3. Discrepancy is sub-multiplicative, i. e.,

$$
\operatorname{disc}(\mathcal{G} \times \mathcal{H}) \leq \operatorname{disc}(\mathcal{G}) \operatorname{disc}(\mathcal{H})
$$

Proof of Theorem 3.1: It follows from Definition 1.1 that the hypergraph of $d$-dimensional arithmetic progressions is nothing else than the $d$-fold direct product of the hypergraph of one-dimensional arithmetic progressions. Using optimal colorings for any of the factors of the hypergraph of $d$-dimensional arithmetic progressions arising from the theorem of Matoušek and Spencer [MS96], Theorem 3.3 implies Theorem 3.1.

A problem of some interest on its own is to decide if or to what extent the discrepancy of $\mathcal{G} \times \mathcal{H}$ can be smaller than the product $\operatorname{disc}(\mathcal{G}) \operatorname{disc}(\mathcal{H})$. The case of arithmetic progressions might suggest equality, but this is not the case, as the following examples show:

Example 1: The hypergraph of two-element subsets of a three-element set $\mathcal{G}=\left([3],\left(\begin{array}{c}{[3]} \\ 2\end{array}\right)\right)$ has discrepancy two (one color class has at least two elements, i.e., it contains a monochromatic two-set). The direct product $\mathcal{G} \times \mathcal{G}$ can be colored in a way that there is no monochromatic rectangle: $\chi(i, i):=1$ and $\chi(i, j):=-1$ for $i, j \in[3], i \neq j$. So $\operatorname{disc}(\mathcal{G} \times \mathcal{G}) \leq 2<4=\operatorname{disc}(\mathcal{G})^{2}$. (Easy to see if we visualize $\mathcal{G} \times \mathcal{G}$ like that: The vertices form a $3 \times 3$-grid, the hyperedges consist of the corners of the rectangles having axis-parallel edges. All these rectangles have one or two points on the diagonal of the grid, thus having discrepancy two or zero with respect to $\chi$.)

Looking at examples like this one might ask whether the discrepancy of a direct product is at least the discrepancy of its factors, or in an even weaker form we ask, whether the discrepancy of a direct product of two hypergraphs of nonzero discrepancy has discrepancy greater than 0 . In general this is not true:

Example 2: Let $\mathcal{G}$ be the hypergraph

$$
(\{1, \ldots, 7\},\{\{1,2\},\{1,3\},\{1,4\},\{1,5\},\{2,3,4,5,6,7\}\})
$$

as depicted in Figure 1.

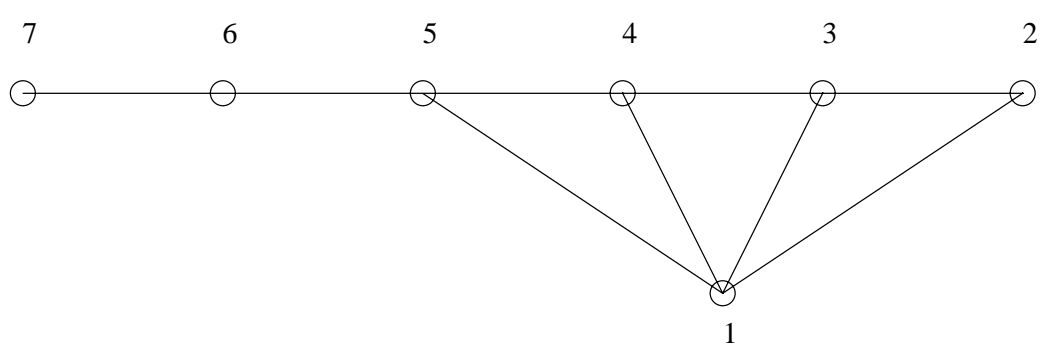

Figure 1: Example 2 
$\mathcal{G}$ does not have discrepancy 0 ; if so, the points $2,3,4$ and 5 were in the same color class leaving the edge $E=\{2,3,4,5,6,7\}$ imbalanced.

The hypergraph $\mathcal{G} \times \mathcal{G}$ however has discrepancy 0. The coloring depicted in Figure 2 does the job.

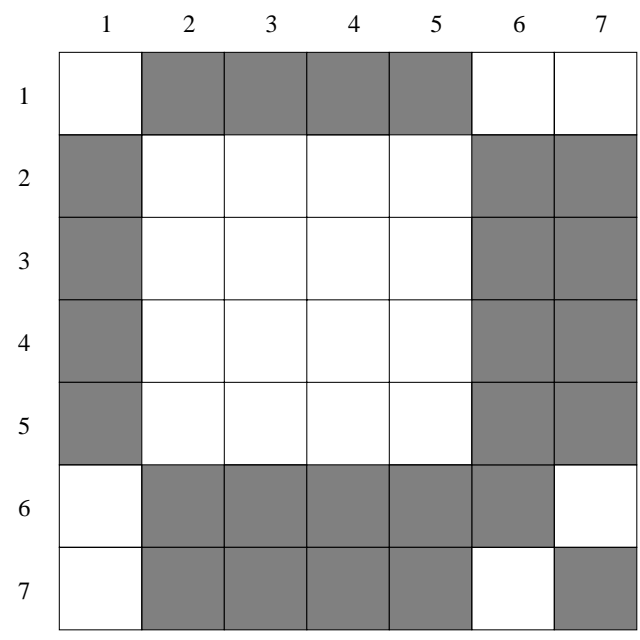

Figure 2: A coloring of $\mathcal{G} \times \mathcal{G}$ with discrepancy 0 


\section{Some Special Cases}

In this section, we investigate some related problems.

\subsection{Symmetric Arithmetic Progressions}

First we consider $d$-dimensional arithmetic progressions that are the product of just one arithmetic progression (we call them symmetric), i. e., our hypergraph $\mathcal{H}_{S}$ is defined by

$$
\mathcal{H}_{S}=\left([N]^{d},\left\{\prod_{i=1}^{d} A \mid A \text { arithmetic progression }\right\}\right) .
$$

At the workshop of the graduate school ,Algorithmische Diskrete Mathematik" in Berlin in April 1997 Walter Deuber asked about the bounds for the discrepancy of this hypergraph.

Note that this is a special case of our general problem in the sense that $\mathcal{H}_{S} \subset \mathcal{H}$. This shows $\operatorname{disc}\left(\mathcal{H}_{S}\right) \leq c N^{\frac{d}{4}}$, but actually we have a much stronger result.

Theorem 4.1. There is a constant $C$ independent of $d$ and $N$ such that

$$
\operatorname{disc}\left(\mathcal{H}_{S}\right) \leq C N^{\frac{1}{4}}
$$

We give a general solution.

Let $\mathcal{H}=(X, \mathcal{E})$ denote a hypergraph. Set $\mathcal{E}_{\text {sym }}^{d}:=\left\{E^{d} \mid E \in \mathcal{E}\right\}$. We call $\mathcal{H}_{\text {sym }}^{d}:=$ $\left(X^{d}, \mathcal{E}_{\text {sym }}^{d}\right)$ the $d$-fold symmetric direct product of $\mathcal{H}$. We have

\section{Theorem 4.2.}

$$
\operatorname{disc}\left(\mathcal{H}_{\text {sym }}^{d}\right) \leq \operatorname{disc}(\mathcal{H})
$$

Proof. Let $D:=\{(x, \ldots, x) \mid x \in X\}$ be the diagonal of $X^{d}$. For $x \in X^{d} \backslash D$ set

$$
a(x):=\min \left\{i \mid x_{i} \neq x_{i+1}\right\} .
$$

Define $f: X^{d} \rightarrow X^{d}$ by

$$
f(x)_{i}:= \begin{cases}x_{a(x)+1} & \text { if } x \notin D, i \leq a(x) \\ x_{1} & \text { if } x \notin D, i=a(x)+1 . \\ x_{i} & \text { otherwise }\end{cases}
$$

Note that $f(f(x))=x$ for all $x \in X^{d}$, so $f$ is a bijection. For all $x \in X^{d} \backslash D$ the $f$-orbit $O_{f}(x)$ of $x$ has order 2 and consists of $x$ and $f(x)$. Further we have

$$
\left\{x_{i} \mid i \in[d]\right\}=\left\{f(x)_{i} \mid i \in[d]\right\},
$$


and thus $f$ leaves the hyperedges of $\mathcal{H}_{\text {sym }}^{d}$ invariant.

Pick an optimal coloring $\chi_{\mathcal{H}}$ of $\mathcal{H}$. Choose a system $R$ of representatives of the $f$-orbits in $X^{d} \backslash D$, i. e., for all $x \in X^{d} \backslash D$ either $x$ or $f(x)$ lies in $R$. Define $\chi: X^{d} \rightarrow\{-1,1\}$ by

$$
\chi(x):= \begin{cases}-1 & \text { if } x \in R \\ \chi_{\mathcal{H}}(v) & \text { if } x=(v, \ldots, v) \in D . \\ 1 & \text { otherwise }\end{cases}
$$

Let $E \in \mathcal{E}$. From the properties of $f$ and $R$ we deduce $\left|E^{d} \cap R\right|=\left|f\left(E^{d} \cap R\right)\right|=$ $\left|f\left(E^{d}\right) \cap f(R)\right|=\left|E^{d} \cap\left(X^{d} \backslash D \backslash R\right)\right|=\left|E^{d} \backslash D \backslash R\right|$. So we have

$$
\sum_{x \in E^{d}} \chi(x)=\sum_{x \in E^{d} \cap R}-1+\sum_{x \in E^{d} \cap D} \chi(x)+\sum_{x \in E^{d} \cap f(R)} 1=\sum_{v \in E} \chi_{\mathcal{H}}(v),
$$

and this proves the theorem.

Unfortunately, we do not know very much about lower bounds. For the general case, nothing can be said, as is obvious from Example 2 in Section 3. In the special case of arithmetic progressions, it is not possible to use the circle-method in the way of Section 2, because the convolution and Fourier-transform take place on different groups, namely $\mathbb{Z}^{d}$ and the diagonal of $\mathbb{Z}^{d}$. Thus we do not have $\left\|\widehat{\chi_{F} * \eta_{\delta}}\right\|_{2}^{2}=\left\|\widehat{\chi}_{F} \cdot \widehat{\eta}_{\delta}\right\|_{2}^{2}$, i. e., we are not able to separate the coloring from the characteristic function, which was one main step in the proof of Theorem 2.1.

\subsection{Arithmetic Progressions on Lines}

Another generalization of one-dimensional arithmetic progressions are one-dimensional arithmetic progressions in the $[N]^{d}$-lattice.

Let $l \in\{1, \ldots, N\}, u \in[N]^{d}$ and $v \in\{-(N-1), \ldots, N-1\}^{d}$ such that $u+(l-1) v \in[N]^{d}$. Then we call $A_{l, u, v}:=u+[l] v=\{u+j v \mid j \in[l]\}$ an arithmetic progression on a line. For the hypergraph of all arithmetic progressions on lines $\mathcal{H}_{L}^{d}$, in the mean-time Valko [Val02] has found sharper bounds than in [Weh97]. He proved a lower bound of $\Omega\left(N^{d /(2 d+2)}\right)$ and an upper bound of $O\left(N^{d /(2 d+2)}\left(\log ^{\frac{5}{2}} N\right)\right.$.

Yet, if we allow only "a few" lines, we can determine the discrepancy quite well. First let us consider only lines parallel to the axes. Then from the product coloring argument in Section 3, the following is obvious.

Corollary 4.3. Let $\mathcal{E}_{L_{0}}^{d}=\left\{A_{l, u, v} \mid \exists ! i \in\{1, \ldots, d\}: v_{i} \neq 0\right\}$ and let $\mathcal{H}_{L_{0}}^{d}:=\left([N]^{d}, \mathcal{E}_{L_{0}}^{d}\right)$ be the hypergraph under consideration. Then

$$
\operatorname{disc}\left(\mathcal{H}_{L_{0}}^{d}\right)=\Theta\left(N^{\frac{1}{4}}\right) .
$$


We may also add any constant number of lines and still get a result sharp up to polylogarithmic factors:

Theorem 4.4. For all $C, d \in \mathbb{N}$ exist constants $c, c^{\prime}>0$ such that for all $V \subseteq[N]^{d}$, $|V|=C$ and $\mathcal{H}_{L, V}^{d}:=\left([N]^{d},\left\{A_{l, u, v} \mid v \in V\right\} \cup \mathcal{E}_{L_{0}}^{d}\right)$ we have

$$
c N^{\frac{1}{4}} \leq \operatorname{disc}\left(\mathcal{H}_{L, V}^{d}\right) \leq c^{\prime} N^{\frac{1}{4}} \log ^{\frac{5}{2}} N
$$

Proof. The lower bound is clear. The upper bound is (apart from a constant) the same as the upper bound that J. Beck achieved for 1-dimensional arithmetic progressions [Bec81]. Like there we can decompose any arithmetic progression into $O(\log N)$ canonical arithmetic progressions (this is a one-dimensional phenomenon). Therefore

$$
\operatorname{disc}\left(\mathcal{H}_{L, C}^{d}\right) \leq c_{0} \log N \operatorname{disc}\left(\mathcal{H}_{C}\right)
$$

where $\mathcal{H}_{C}$ is the hypergraph of the canonical sets and $c_{0}$ a constant independent on $N$. Similarly, all degree problems can be bounded by the respective one-dimensional result times $C$, hence for a suitable constant $c_{1}$ we also get $\operatorname{deg}\left(\left\{A \in \mathcal{H}_{C}:|A| \geq c_{1} \sqrt{N}\right\}\right) \leq$ $c_{1} \sqrt{N}$. Thus a theorem of Beck [Bec81] yields $\operatorname{disc}\left(\mathcal{H}_{C}\right) \leq c_{2} N^{\frac{1}{4}} \log ^{\frac{3}{2}} N$ (all constants independent of $N$ ).

\subsection{Arithmetic Progressions in $\mathrm{N}$}

There are also some results on the discrepancy of the hypergraph of all finite arithmetic progressions on the set of all non-negative integers $\mathbb{N}$. An easy consequence of Roth's lower bound proof can be found, e. g., in [BS95]:

Corollary 4.5. Given any 2 -coloring $\chi: \mathbb{N} \rightarrow\{-1,+1\}$ of the non-negative integers, then for infinitely many values of $\delta$ there is a (finite) arithmetic progression $A$ of difference $\delta$ such that

$$
|\chi(A)|>c \sqrt{\delta}
$$

for an absolute constant $c$.

The so far best upper bound for the discrepancy in terms of the difference was shown by J. Beck and J. Spencer [BS84]:

Theorem 4.6. There is a constant $c_{0}$ such that the following holds: Let $n$ be a positive integer. Then there exists a 2 -coloring $\chi: \mathbb{N} \rightarrow\{-1,+1\}$ such that for any arithmetic progression $A$ of difference $\delta \leq n$ and of arbitrary length

$$
|\chi(A)|<c_{0} \sqrt{\delta} \log ^{3.5} n .
$$


We will show an analogous result in the $d$-dimensional case. Let $A_{l, a, \delta}:=a+\delta[l]$ denote the arithmetic progression with starting point $a$, difference $\delta$ and length $l$. For $a, \delta, l \in \mathbb{N}^{d}$ set $A_{l, a, \delta}:=\prod_{i=1}^{d} A_{a_{i}, \delta_{i}, l_{i}}$. Write $\delta>k(\operatorname{resp} . \delta \leq k)$ to express that all components $\delta_{i}$ of $\delta$ are greater than $k$ (resp. less or equal than $k$ ).

Theorem 4.7. For any 2-coloring $\chi: \mathbb{N}^{d} \rightarrow\{-1,+1\}$ and every vector $k \in \mathbb{N}^{d}$ there exists a d-dimensional arithmetic progression $A_{l, a, \delta}$ such that $\delta>k$ and

$$
\left|\chi\left(A_{l, a, \delta}\right)\right|>\pi^{-d} \sqrt{\delta_{1} \ldots \delta_{d}}
$$

Conversely, for any positive integer $n$ there exists a 2 -coloring $\chi: \mathbb{N}^{d} \rightarrow\{-1,+1\}$ such that for any arithmetic progressions $A_{l, a, \delta}$ of difference $\delta \leq n$ and of arbitrary length and starting point

$$
\left|\chi\left(A_{l, a, \delta}\right)\right|<c_{0}^{d} \sqrt{\delta_{1} \ldots \delta_{d}} \log ^{3.5 d} n .
$$

Proof. For the upper bound, the product coloring argument again solves the problem. Let $\chi$ and $k$ be given. Define a $2-$ coloring $\chi_{k}$ of $\mathbb{N}^{d}$ by

$$
\chi_{k}(x):=\chi(k x)
$$

for all $x \in \mathbb{N}^{d}$ (where $\left.k x:=\left(k_{i} x_{i}\right)_{i=1}^{d}\right)$. Choose an integer $N>\|k\|_{\infty}^{2}$. From the proof of Theorem 2.1 we have the existence of vectors $l, \delta^{\prime} \in \Delta=\{1, \ldots, \sqrt{N}\}^{d}$ and $a^{\prime} \in[N]^{d}$ such that

$$
\left|\chi_{k}\left(A_{l, a^{\prime}, \delta^{\prime}}\right)\right|>\pi^{-d} N^{\frac{d}{4}} \geq c \sqrt{\delta_{1}^{\prime} \ldots \delta_{d}^{\prime}}
$$

From $\chi_{k}\left(A_{l, a^{\prime}, \delta^{\prime}}\right)=\chi\left(A_{l, k a^{\prime}, k \delta^{\prime}}\right)$ we see that $A_{l, k a^{\prime}, k \delta^{\prime}}$ is an arithmetic progression satisfying our needs.

\section{Discussion}

This article determines the discrepancy of cartesian products of arithmetic progressions. A related problem remains open: For the symmetric arithmetic progressions a lower bound is missing. Our feeling is that if both the dimension of the grid and the hyperedges are raised from one to $d$, then everything stays fine, but if we change just one dimension, then things get quite difficult.

Also, the problem of a polynomial-time construction of good colorings for arithmetic progressions remains open. For arbitrary hypergraphs this is a $N P$-hard problem. At the moment, there is not much hope even for the hypergraph of arithmetic progressions, because the existence of optimal colorings is proved via the pigeonhole principle. 


\section{Acknowledgments}

The authors are grateful to J. Matoušek, H. J. Prömel and J. Spencer for their interest and helpful discussions on the subject of this article. The first author would like to thank N. Linial and J. Spencer for a fruitful discussion at the discrepancy workshop in Kiel 1998, who suggested the generalization of our product coloring argument in Section 3 to multi-colorings.

\section{References}

[Bec81] J. Beck. Roth's estimate of the discrepancy of integer sequences is nearly sharp. Combinatorica, 1:319-325, 1981.

[BS84] J. Beck and J. Spencer. Well distributed 2-colorings of integers relative to long arithmetic progressions. Acta Arithm., 43:287-298, 1984.

[BS95] J. Beck and V. T. Sós. Discrepancy theory. In R. Graham, M. Grötschel, and L. Lovász, editors, Handbook of Combinatorics, pages 1405-1446. Elsevier, 1995.

[DS03] B. Doerr and A. Srivastav. Multicolour discrepancies. Combinatorics, Probability and Computing, 12:365-399, 2003.

[GRS90] R. L. Graham, B. L. Rothschild, and J. H. Spencer. Ramsey Theory. John Wiley \& Sons Inc., New York, 1990.

[MS96] J. Matoušek and J. Spencer. Discrepancy in arithmetic progressions. J. Amer. Math. Soc., 9:195-204, 1996.

[Rad33] R. Rado. Verallgemeinerung eines Satzes von van der Waerden mit Anwendung auf ein Problem der Zahlentheorie. Sitzungsber. Preuss. Akad. Wiss., Phys.Math. Kl., 16/17:589-596, 1933.

[Rot64] K. F. Roth. Remark concerning integer sequences. Acta Arithmetica, 9:257-260, 1964.

[Rud62] W. Rudin. Fourier Analysis on Groups. John Wiley and Sons, New York, 1962.

[Sár74] A. Sárközy. In P. Erdős and J. Spencer, editors, Probabilistic Methods in Combinatorics. Akadémia Kiadó, Budapest, 1974.

[Val02] B. Valkó. Discrepancy of arithmetic progressions in higher dimensions. Journal of Number Theory, 92:117-130, 2002.

[vdW27] B. L. van der Waerden. Beweis einer Baudetschen Vermutung. Nieuw Arch. Wsk., 15:212-216, 1927. 
[Weh97] P. Wehr (Knieper). The Discrepancy of Arithmetic Progressions. 1997. Dissertation, Institut für Informatik, Humboldt-Universität zu Berlin. 